\title{
炔基吡喃酮的环异构化合成酚类化合物
}

\author{
关正辉* 祝海涛 \\ (西北大学化学与材料科学学院 西安 710127)
}

\section{Deconstructive Reorganization of Alkynyl Pyranones for the Synthesis of Phenols}

\author{
Guan, Zhenghui* Zhu, Haitao \\ (Department of Chemistry \& Materials Science, Northwest University, Xi'an 710127)
}

酚类化合物在医药、农药以及日用化学品等领域具 有重要的应用, 其中羟基苯并呋喃是许多天然产物和生 物活性分子的结构骨架. 如 Scheme 1 所示, 天然产物 Coumestrol 具有抗癌、调节精神紊乱等多种生物活性; Liphagal 是人类 PI3K 信号通路的调节子, 有望用于自身 免疫疾病、心血管疾病和癌症等的治疗; BNC1005 可抑 制微管蛋白的聚集, 防止肿瘤细胞扩散, 并已进入治疗 血管瘤的 II 期临床试验 ${ }^{[1 \sim 3]}$. 然而, 由于酚羟基具有一 定的酸性且易被氧化, 羟基苯并呋喃的合成仍是有机合 成中具有挑战性的研究课题.

近年来, 华南理工大学祝诗发课题组 ${ }^{[4]}$ 利用炔基吡 喃酮的环异构化过程合成酚类化合物方面取得了重要 进展. 2018 年, 他们以天然麦芽酚(Maltol)和曲酸(Kojic acid)为原料, 发展了吡喃酮的新型去芳构化串联克莱森
重排反应，实现了多取代水杨醛类化合物的快速构建， 并以此方法为关键步骤合成了天然产物棉酚(Gossypol) 和半棉酚(Hemigossypol)(Scheme 2). 通过对反应机理的 深入研究和对反应条件的调控, 他们 ${ }^{[5]}$ 进而实现了含酚 羟基多环骨架的高效合成, 同时还提出了新型 12 电子 Mobius 芳香过渡态反应模型.

最近, 针对羟基苯并呋喃合成中的挑战性, 基于他 们提出的氧化态转移策略, 祝诗发课题组 ${ }^{[6]}$ 发展了路易 斯酸催化炔基吡喃酮的环异构化新反应. 该反应以炔基 麦芽酚和炔基曲酸等为原料, 以三氟甲磺酸铟为催化 剂、四氢呋喃为溶剂, 在 $60 \sim 100{ }^{\circ} \mathrm{C}$ 下即可高效进行. 机理研究表明, $\alpha$-炔基吡喃酮首先在铟盐的催化下发生 5-endo-dig 环化得到关键活性中间体呋喃并吡喃鎓盐, 该中间体经水解或醇解、分子内羟醛缩合和脱水消除等

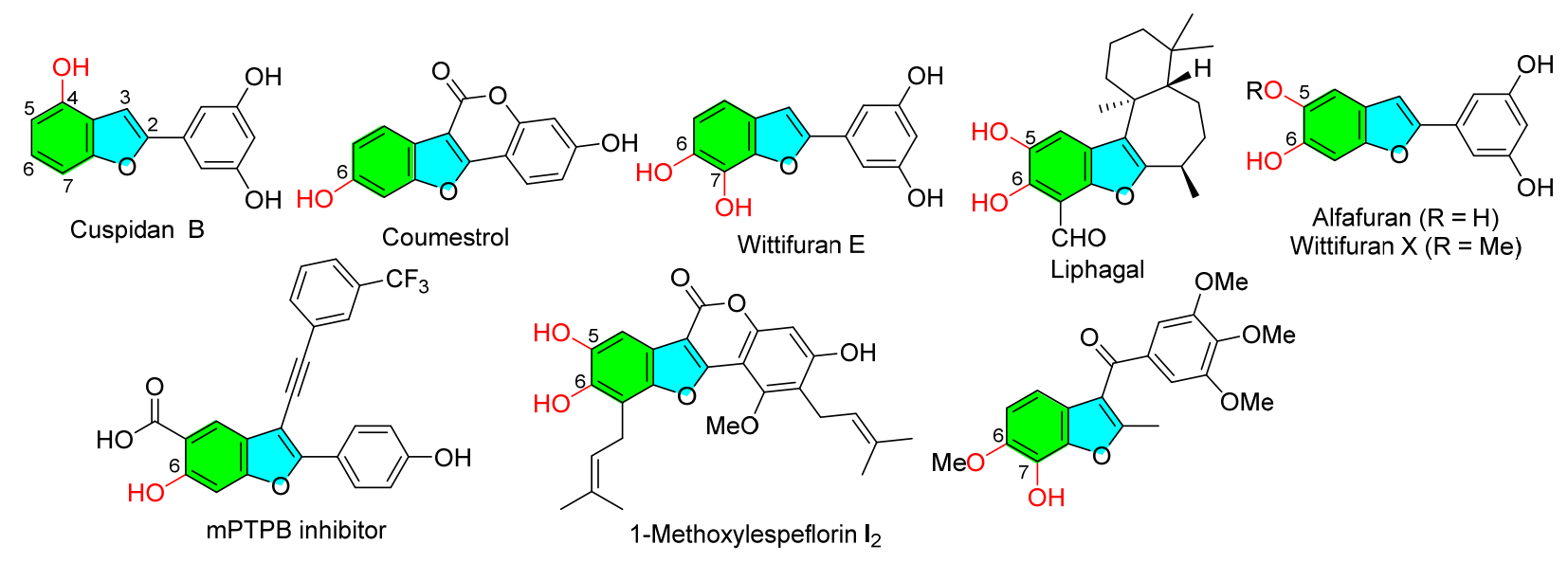

图式 1 具有多羟基苯并呋喃结构的天然产物和候选药物例举

Scheme 1 polyphenolic compounds with biological activities

* Corresponding author. E-mail: guanzhh@nwu.edu.cn. Published online March 23, 2020. 


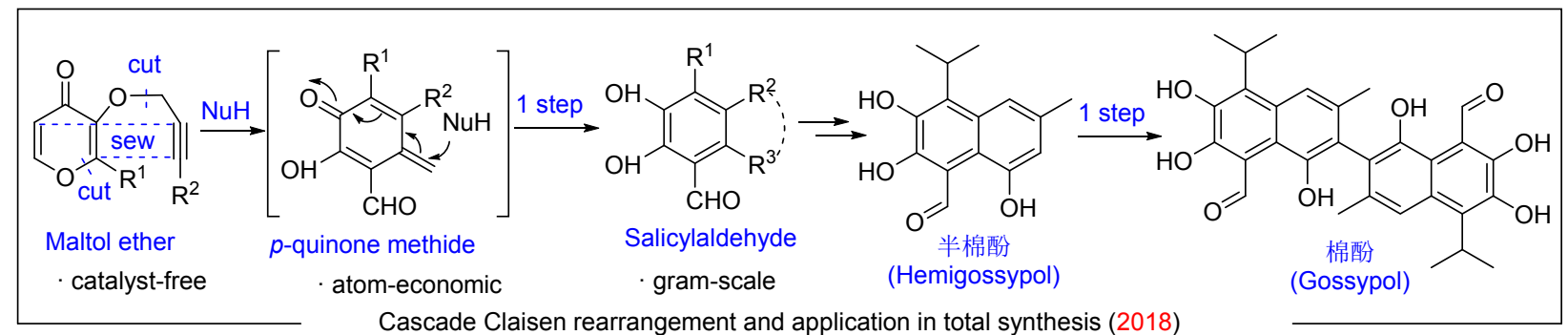

\begin{tabular}{|c|c|c|c|c|c|}
\hline $\begin{array}{l}\text { Natural product } \\
\text { - Price: } \$ 14 / \mathrm{kg} \\
\text { - Food flavor } \\
\text { - Perfume }\end{array}$ & 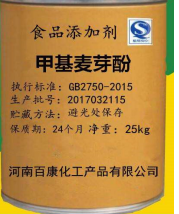 & 麦芽酚 (Maltol) & 曲酸 (Kojic acid) & 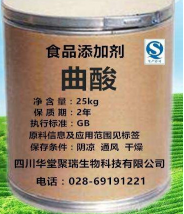 & $\begin{array}{l}\text { - Natural product } \\
\text { - Price: } \$ 17 / \mathrm{kg} \\
\cdot \text {. Food flavor } \\
\cdot \text { Cosmetics }\end{array}$ \\
\hline
\end{tabular}

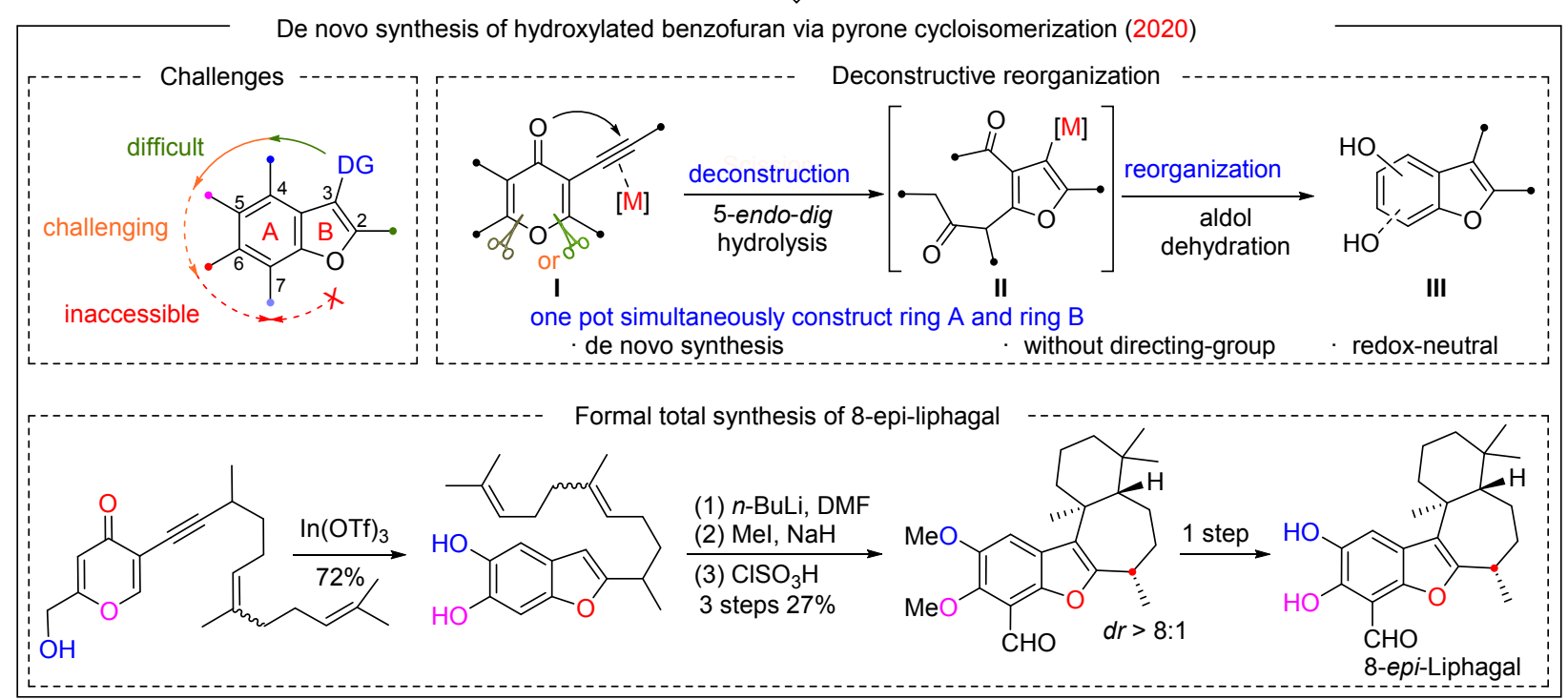

图式 2 麦芽酚和曲酸的转化及炔基吡喃酮环异构化合成多酚

Scheme 2 Synthesis of polyphenol from pyranones

转化, 最终生成环异构化产物多羟基苯并呋喃 III (Scheme 2).

由于温和的反应条件和良好的官能团兼容性，该方 法可以实现 4-, 5-，6-，7-单或双羟基取代苯并呋喃的高 效、高选择性合成(共 72 个实例), 为羟基苯并呋喃的化 学合成提供了直接和通用的新方法. 以此方法学为基 础, 作者还实现了 10 个多羟基苯并呋喃类活性天然产 物的集群式全合成. 另外, 特别需要指出的是, 作者还 以自己发展的新方法为关键步骤，以 $72 \%$ 的产率合成了 复杂天然产物 8-epi-Liphagal 的关键中间体 2-取代 5,6二羟基苯并呋喃, 进而实现了该天然产物的形式全合成 (Scheme 2).

总之, 祝诗发课题组针对多羟基酚合成中的挑战, 利用氧化态转移策略, 巧妙地发展了路易斯酸催化吡喃 酮的环异构化合成多羟基苯并呋喃的新反应. 该反应以
易得的天然麦芽酚和曲酸为原料, 具有条件温和、官能 团兼容性好、底物适用性广和原子经济性高等优点，为 多羟基苯并呋喃类天然产物和生物活性化合物的化学 合成提供了新颖、绿色和高效的方法.

\section{References}

[1] Thuy, N. T. T.; Lee, J.-E.; Yoo, H.; Cho, M. N. J. Nat. Prod. 2019, $82,3025$.

[2] Flynn, B. L.; Gill, G. S.; Grobelny, D. W.; Chaplin, J. H.; Paul, D.; Leske, A. F.; Lavranos, T. C.; Chalmers, D. K.; Charman, S. A.; Kostewicz, E.; Shackleford, D. M.; Morizzi, J.; Hamel, E.; Jung, M. K.; Kremmidiotis, G. J. Med. Chem. 2011, 54, 6014.

[3] Hajduk, P. J. J. Med. Chem. 2006, 49, 6972.

[4] Cao, T.; Kong, Y.; Luo, K.; Chen, L.; Zhu, S. Angew. Chem., Int. Ed. 2018, 57, 8702 .

[5] Cao, T.; Chen, L.; Zhu, S. Org. Lett. 2019, 21, 90.

[6] Zhang, L.; Cao, T.; Jiang, H.; Zhu, S. Angew. Chem., Int. Ed. 2020, 59,4670 .

(Zhao, C.) 